products indicated that $\mathrm{A} 2071 \mathrm{G}$ and $\mathrm{A} 2072 \mathrm{G}$ transitions in the 23S rRNA gene was the major mutations. No mutation was found in gyrA QRDR. Three patients (13.6\%), including 1 couple, carried G248T (S83I) mutation in parC QRDR. The female of the couple also carried a parC G259C (D87H) mutant group. Two individuals (a couple) carried organisms that had markers for both macrolide and fluoroquinolone resistance.

Conclusion This is the first study based in the USA to document both fluoroquinolone and macrolide resistance in $\mathrm{MG}$ using a molecular-based assay. We plan to genotype MG directly from the clinical specimens to investigate the genetic relatedness of antimicrobial-susceptible and resistant-MG, as well as the concordance of MG in couples.

\section{P3.139 ENHANCED SURVEILLANCE OF INFECTIOUS SYPHILIS AND THE CASCADE-OF-CARE AMONG HIV-POSITIVE AND HIV-NEGATIVE MEN WHO HAVE SEX WITH MEN IN BRITISH COLUMBIA, CANADA}

${ }^{1}$ Lukac $\mathrm{Cd},{ }^{1} \mathrm{~T}$ Consolacion, ${ }^{1} \mathrm{~B}$ Brownrigg, ${ }^{1} \mathrm{H}$ Jiang, ${ }^{1} \mathrm{C}$ Prescott, ${ }^{1} \mathrm{Bccdc}$ Syphilis Nurses, ${ }^{2} \mathrm{M}$ Gilbert, ${ }^{2} \mathrm{~T}$ Grennan, ${ }^{2} \mathrm{~J}$ Wong. ${ }^{1}$ British Columbia Centre for Disease Control, Vancouver, Canada; ${ }^{2}$ British Columbia Centre for Disease Control, University of British Columbia, Vancouver, Canada

\subsection{6/sextrans-2017-053264.374}

Introduction From 2010 to 2015, the incidence of infectious syphilis (primary, secondary, and early-latent) has increased 5fold in British Columbia (BC). In response, the BC Centre for Disease Control (BCCDC) enhanced surveillance for syphilis to characterise sexual/social networks driving the epidemic, and to monitor the risk of HIV transmission. Here we communicate indicators developed from the provincial enhanced surveillance system.

Methods In BC, management of syphilis - including partner notification - is centralised, and coordinated by the BCCDC. In January 2016, a new workflow was implemented to systematically collect and analyse data on HIV co-infection, viralload and partners. New indicators were developed along a cascade-of-care framework for case and partner care.

Results From January to September 2016, 581 syphilis cases were diagnosed in BC; 491 (84\%) were among men who have sex with men (MSM). Of these, 201 (41\%) were HIVpositive and $268(55 \%)$ were HIV-negative. Three-quarters of HIV-positive MSM had undetectable viral loads. 149 (74\%) of HIV-positive MSM and 137 (51\%) of HIV-negative MSM were diagnosed during the early-latent stage. For both groups, $96 \%$ of cases were treated within 30 days of syphilis testing. Of the 201 HIV-positive MSM, 141 (70\%) discussed partners with public health nurses and together reported 1270 partners $(65 \%$ anonymous, $35 \%$ notifiable) or 9.0 partners/case (range:0-214). Of the 268 HIV-negative MSM, 215 (80\%) discussed partners and reported 1806 partners $(51 \%$ anonymous, 49\% notifiable), or 8.4 partners/case (range:1-200).

Conclusion A greater proportion of HIV-positive MSM were diagnosed with syphilis during the asymptomatic early-latent stage, which may be due to routine syphilis screening. However, a lower proportion of HIV-positive MSM with syphilis co-infection were engaged with public health for partner notification, and report a lower proportion of notifiable partners, compared to MSM with syphilis only. Strategies to engage HIV-positive MSM in partner care would strengthen the public health response to syphilis.

\section{P3.140 HIGH PREVALENCE OF ASYMPTOMATIC SEXUALLY TRANSMITTED INFECTIONS AMONG HIV-INFECTED PREGNANT WOMEN IN SOUTH AFRICA}

${ }^{1}$ Maanda Mudau, 'Andrew Medina-Marino, 'Lindsey De Vos, 'Dawie Olivier, ${ }^{2}$ Dvora Joseph Davey, ${ }^{3}$ Remco P Peters, ${ }^{3}$ James A Mcintyre, ${ }^{4}$ Xiaoyan Wang, ${ }^{4}$ Jeffrey D Klausner. ${ }^{1}$ Foundation for Professional Development, Pretoria, South African Republic; ${ }^{2}$ University of Cape Town - Cape Town, South African Republic; ${ }^{3}$ ANOVA Health Institute, Johannesburg, South African Republic; ${ }^{4}$ UCLA, Los Angeles, USA

\subsection{6/sextrans-2017-053264.375}

Introduction: Chlamydia trachomatis (CT), Neisseria gonorrhoea (NG) and Trichomonas vaginalis (TV) are major contributors to the global burden of disease. During pregnancy, these Sexually Transmitted Infections (STIs) may lead to complications including intrauterine death and preterm delivery, and may facilitate mother-to-child-transmission (MTCT) of HIV. Treatment of these infections is suboptimal due to the syndromic approach to diagnosis and management. We conducted an analysis to determine the prevalence of CT, NG and TV among HIV infected pregnant women and prevalence of symptoms among those infected.

Methods HIV-infected pregnant women accessing antenatal care (ANC) services for the first time for their current pregnancy were invited to take part in the study. Participants were interviewed using a questionnaire and asked to self-collect two vaginal swab specimens to test for CT, NG and TV. Tests were done by nurses in the clinic using the Xpert CT/NG and Xpert TV [Cepheid, Sunnyvale, CA].

Results Overall 192 women were tested for CT, NG and TV, of whom 52.1\% (100/192) were had at least one infection. CT had the highest prevalence (40.6\%), followed by TV $(26.6 \%)$, while NG had the lowest prevalence $(7.3 \%)$. Based on self-reported symptoms, $78 \%$ of infected individuals were asymptomatic; 68\% were asymptomatic when using clinicianobserved symptoms. Using a combination of both, 58\% were asymptomatic. Individuals infected with NG were most likely to be symptomatic $(73.3 \%)$, followed by TV (51.9\%), then CT (43.1\%) using self-report and clinician observation.

Conclusion This analysis shows that HIV-infected pregnant women have a high burden of three curable STIs that are associated with adverse pregnancy outcomes and may have links to HIV MTCT. Most infected women were asymptomatic when using either symptom self-reporting or clinician observation or a combination of both, highlighting the limitations of the symptoms-based approach to STI diagnosis. Therefore, the use of definitive POC tests for routine STI screening must be considered in this setting.

\section{P3.141 CHLAMYDIA TRACHOMATIS, NEISSERIA GONORRHOEAE AND TRICHOMONAS VAGINALIS COLONISATION AMONG HIV-EXPOSED NEONATES IN SOUTH AFRICA}

${ }^{1}$ Maanda Mudau, ${ }^{1}$ Andrew Medina-Marino, 'Lindsey De Vos, 'Dawie Olivier, ${ }^{2}$ Dvora Joseph Davey, ${ }^{3}$ Remco P Peters, ${ }^{4}$ Kristina Adachi, ${ }^{3}$ James A Mcintyre, ${ }^{4}$ Xiaoyan Wang, ${ }^{4}$ Jeffrey D Klausner. ${ }^{1}$ Foundation for Professional Development, Pretoria, South African Republic; ${ }^{2}$ University of Cape Town - Cape Town, South African Republic; ${ }^{3}$ ANOVA Health Institute, Johannesburg, South African Republic; ${ }^{4}$ UCLA, Los Angeles, USA

\subsection{6/sextrans-2017-053264.376}

Introduction Sexually transmitted infections (STIs) such as Chlamydia trachomatis (CT), Neisseria gonorrhoeae (NG) and Trichomonas vaginalis (TV) are associated with adverse 